\title{
High-content behavioral profiling reveals neuronal genetic network modulating Drosophila larval locomotor program
}

\author{
Boanerges Aleman-Meza, Mario Loeza-Cabrera, Omar Peña-Ramos, Michael Stern and Weiwei Zhong* ${ }^{*}$
}

\begin{abstract}
Background: Two key questions in understanding the genetic control of behaviors are: what genes are involved and how these genes interact. To answer these questions at a systems level, we conducted high-content profiling of Drosophila larval locomotor behaviors for over 100 genotypes.

Results: We studied 69 genes whose C. elegans orthologs were neuronal signalling genes with significant locomotor phenotypes, and conducted RNAi with ubiquitous, pan-neuronal, or motor-neuronal Gal4 drivers. Inactivation of 42 genes, including the nicotinic acetylcholine receptors $n A C h R a 1$ and $n A C h R a 3$, in the neurons caused significant movement defects. Bioinformatic analysis suggested 81 interactions among these genes based on phenotypic pattern similarities. Comparing the worm and fly data sets, we found that these genes were highly conserved in having neuronal expressions and locomotor phenotypes. However, the genetic interactions were not conserved for ubiquitous profiles, and may be mildly conserved for the neuronal profiles. Unexpectedly, our data also revealed a possible motor-neuronal control of body size, because inactivation of Rdl and Gao in the motor neurons reduced the larval body size. Overall, these data established a framework for further exploring the genetic control of Drosophila larval locomotion.
\end{abstract}

Conclusions: High content, quantitative phenotyping of larval locomotor behaviours provides a framework for system-level understanding of the gene networks underlying such behaviours.

Keywords: High-content phenotypic profiling, Genetic interaction network, Larval locomotion, Behaviour, Drosophila melanogaster

\section{Background}

A key challenge in neurobiology is to connect behaviors to neurons to genes. The fruitfly Drosophila melanogaster larva is a powerful model for research in this area. Quantitative studies of the larval locomotor behaviors, such as [1-6], have enabled many discoveries linking genes, neurons, and phenotypes. Recent technologies in high-throughput phenotyping have advanced research in this area from a single gene level to a systems level. For example, a multi-larvae tracking system [7], in combination with optogenetics and a vast collection of Gal4 drivers, have enabled discovery of the neuronal circuits regulating behaviors such as peristaltic crawling [8] and decision-making in response to a mechano-stimulus [9].

\footnotetext{
* Correspondence: weiwei.zhong@rice.edu

Department of BioSciences, Rice University, Houston, TX 77005, USA
}

Connecting behaviors to gene networks remains largely unexplored at the systems level even for this relatively simple animal. To understand the gene networks, one must reveal which genes are involved (nodes), and how they interact (edges). The major challenge is how to map genetic interactions. One method to reveal genetic interactions is epistasis analysis [10], which compares double and single mutant phenotypes to identify enhancing and suppressing effects. In Drosophila, this method requires genetic crosses to generate double mutants, and is thus not practical for large-scale mapping of many genes. In such cases, high-content profiling becomes an effective method in mapping genetic interactions. Genes with similar phenotypic profiles are considered interacting. In animals, high-content profiling was successfully applied to discover gene interaction networks regulating 
C. elegans embryogenesis [11], gonad architecture [12], and locomotor behaviors [13, 14].

We have recently developed the imaging system MaggotTracker to analyse multiple parameters of Drosophila larval locomotor behaviors [15]. MaggotTracker tracks a single animal at a high resolution and measures over 20 parameters, enabling high-content profiling of Drosophila phenotypes.

Here we present high-content larval locomotor profiles for 69 genes using the MaggotTracker. These profiles revealed 42 genes whose inactivation in the neurons caused significant movement defects, and 81 genetic interactions among these genes. As we focused on Drosophila genes whose C. elegans orthologs are locomotor genes, this data set also revealed that while many genes are conserved in their involvement in locomotor behaviors and neuronal expression, the genetic interactions were conserved to a much lesser degree. Finally, our data suggested a motor-neuronal control of body length, as inactivation of $R d l$ and $G \alpha o$ in motor neurons caused reduced larval size.

\section{Methods}

\section{Animals}

Fly strains were obtained from the Bloomington Drosophila Stock Center (IN). All stocks were maintained on cornmeal agar following the Bloomington food recipe $(0.8 \%$ yeast, $0.93 \%$ soy flour, $6.79 \%$ yellow cornmeal, $0.8 \%$ agar, $7.1 \%$ Karo light corn syrup, and $0.45 \%$ propionic acid). Animals were cultured at room temperature $\left(\sim 22{ }^{\circ} \mathrm{C}\right)$ for regular stock maintenance. All RNAi strains are listed in Additional file 1: Table S1. The Gal4 strains used in this study were D42-Gal4 [16], elav-Gal4 [17], dilp2-Gal4 [18], tub-Gal4 (Bloomington \#5138), and daGal4 (\#108252) which was provided by the Drosophila Genetic Resource Center.

\section{Genetic crosses}

Males from the UAS-dsRNA strain and virgin females from the Gal4 driver strain were crossed. A strain expressing UAS-dsRNA for mCherry RNAi (stock \# 35785) was used as control. For RNAi strains in which the CyO balancer was present in the stock, the CyO was replaced with CyORoi, and males with smooth eyes (lacking the balancer) were used in the cross. The stock number and RNAi gene for these balanced strains are \#38966, CanA-14F; \#44014, Syb; \#44538, CG1909; \#44539, robo3; \#51049, nAChR 7 ; \#53296, unc-104.

Two RNAi stocks (\#28574, RSG7; \#36746, CG31140) carried the TM3 balancer. In crosses involving these two strains, larvae were recovered after tracking, placed in a 24 well plate containing cornmeal agar (1 larva per well) and left at $20{ }^{\circ} \mathrm{C}$ until they became adult flies. Then each fly was inspected to determine if it carried the balancer.
Videos of the larvae that later showed the stubble phenotype (carrying the TM3 balancer) were discarded.

The default culture temperature for genetic crosses was $25{ }^{\circ} \mathrm{C}$. That is, while parental strains were maintained at room temperature $\left(\sim 22{ }^{\circ} \mathrm{C}\right)$, genetic crosses, which included placing males and females from two different parental strains together, and subsequent larval growth, were kept at $25{ }^{\circ} \mathrm{C}$. If RNAi caused lethality, crosses were kept at $20{ }^{\circ} \mathrm{C}$. For crosses using the $t u b$ Gal4 driver, if $20{ }^{\circ} \mathrm{C}$ still caused lethality, then da-Gal4 driver was used. Similarly, da-Gal4 driver was tested at $25{ }^{\circ} \mathrm{C}$ first and reduced to $20{ }^{\circ} \mathrm{C}$ if there was lethality. At least 3 independent crosses were conducted for each strain. Animals from these crosses were pooled prior to analysis.

\section{Behavioral assay}

Animals were tracked using the MaggotTracker as described [15]. Briefly, animals were placed at $20{ }^{\circ} \mathrm{C}$ for at least $12 \mathrm{~h}$ before third instar wandering larvae were collected using a paintbrush. Animals were examined under a dissecting scope to determine the gender and confirm the absence of food. A larva was then placed on a $10 \mathrm{~cm}$ Petri dish plate containing 1.5\% agar, and left on the plate for at least $30 \mathrm{~s}$ so that they could acclimate to the media. The animal was then tracked for $4 \mathrm{~min}$ using the computer-controlled system. At least 10 males and 10 females were tracked for each genotype. At least 10 control animals (5 males and 5 females) were tracked in every tracking session.

\section{Statistical analysis}

Mutant parameter values were normalized by the mean values of control animals tested in the same experiment. Mutant locomotive profiles were compared with control ones using the Student's t-test. Benjamini-Hochberg procedure [19] was applied to correct multiple comparisons to control the false discovery rate (FDR) below $1 \%$.

\section{Clustering and network visualization}

Gene Cluster 3.0 [20] was used to perform hierarchical clustering with centroid linkage. Java TreeView [21] was used to display the clusters. Network visualization was performed using the software GUESS (graphexploration.cond.org).

\section{A strategy for high-content phenotyping of larval locomotor behaviors}

A research pipeline was designed to conduct highcontent profiling of larval locomotive behaviors for various mutants (Fig. 1a). We have developed an imaging system, MaggotTracker [15], to automatically track a single fruit fly larva, take a video, and extract $20+$ parameters from the video measuring different aspects of larval crawling. Each third instar larva was 


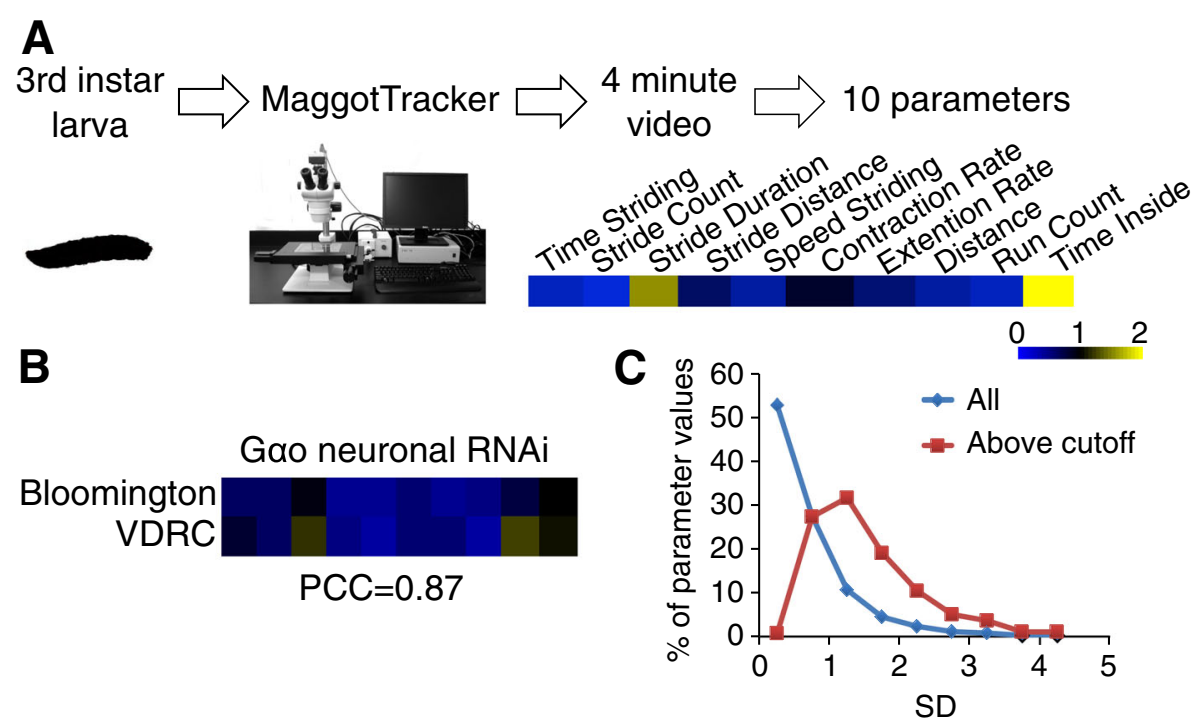

Fig. 1 A strategy to conduct high-content profiling of larval locomotor behaviors. a Third instar larvae of different genotypes were analyzed using the MaggotTracker imaging system. Each larva was tracked and recorded for 4 min. Parameters were extracted from the videos. Mutant values were divided by control values to obtain normalized values. A heatmap was used to display normalized mutant parameter values, with blue, black, yellow indicating mutant values $<_{,}=,>1$, respectively. The same color scale was used for all figures. b Gao neuronal RNAi produced similar phenotypes using UAS-RNAi strains from two stock centers. PCC, Pearson Correlation Coefficient. c Histograms of Z scores from parameter values of all genotypes (All) and parameter values that were considered significantly different than wild-type values (Above cutoff). SD, Standard deviation. Z scores measure how much a mutant value deviate from the wild-type value in SDs

tracked for 4 min. $10+$ males and $10+$ females were tracked for each genotype so that possible sexual dimorphism would be detected.

The phenotypic profiles contained 10 parameters (Table 1) that were selected because they were independent and had similarly low variance [15]. As the parameters were in different units, a normalization process was conducted. Values from mutants were divided by mean values from wild-type animals tracked on the same date to obtain normalized values. This normalization step also reduced day-to-day environmental variations [15]. In the normalized profiles, 1 indicates wild-type value for all parameters; $<1$ and $>1$ indicate lower and higher than wild-type value, respectively.

\section{Results}

\section{High-content behavioral profiles of neuronal signalling genes}

To select candidate genes for testing, we took advantage of a previous high-content phenotyping study of C. elegans locomotor behaviors. We have previously screened 227 worm neuronal signalling genes that had homozygousviable mutants, and found 87 genes with significant locomotor defects [14]. We mapped the D. melanogaster

Table 1 Parameters of the locomotor profiles

\begin{tabular}{lll}
\hline Parameter & Unit & Definition of Parameter \\
\hline Time Striding & $\%$ & Percentage of time when the larva is striding (continuous peristaltic movement). \\
Stride Count & $\mathrm{sec}$ & Total number of strides. \\
Stride Duration & $\mathrm{mm}$ & Time duration of one stride. \\
Stride Distance & $\mathrm{mm} / \mathrm{s}$ & Distance traveled by the center point of the animal during one stride. \\
Speed Striding & $\mathrm{mm} / \mathrm{s}$ & Speed of the center point of the body when the animal is striding. \\
Contraction Rate & $\mathrm{mm} / \mathrm{s}$ & The rate of body length change during the contraction phase of a stride. \\
Extension Rate & The rate of body length change during the extension phase of a stride. \\
Distance & Total distance traveled by the center point. \\
Run Count & Total number of runs. A run is defined as a period when the animal is striding continuously. \\
Time Inside & $\begin{array}{l}\text { Percentage of time the animal is inside the plastic ring that was placed on the outer rim of the } \\
\text { agar plate to prevent the animal from crawling to the edge. }\end{array}$ \\
\hline
\end{tabular}


orthologs of these 87 genes. Among the orthologs, 69 genes have RNAi strains available at the Bloomington stock center (Additional file 1: Table S1). These genes became our candidate genes.

We crossed the UAS-RNAi strains [22] with several GAL4 driver strains to inactivate the gene function in different tissues. A ubiquitous tub-Gal4 driver was used to detect gene function in the entire larvae. If the RNAi resulted in larval lethality, then a weaker ubiquitous driver da-GAL4 was used instead. All genes were also inactivated in the neurons using a pan-neuronal elavGAL4 driver. Selected genes were inactivated using additional GAL4 drivers. For example, for genes that showed strong locomotor phenotypes upon neuronal inactivation, a motor-neuron specific D42-GAL4 driver was used to examine whether these genes function in the motor neurons.

Using the imaging system MaggotTracker (Fig. 1a, also see Method), we obtained high-content locomotive profiles for 128 genotypes of pan-neuronal or ubiquitous RNAi after analysing over 3600 videos of individual animals (Additional file 2: Table S2). Among the 69 genes we tested, excluding those whose RNAi caused larval lethality, neuronal RNAi profiles were obtained for 68 genes, and ubiquitous RNAi profiles were obtained for 60 genes. Upon neuronal RNAi, 42 genes showed significant larval locomotor defect in at least one parameter (FDR < 0.05); upon ubiquitous RNAi, 37 genes showed significant locomotor phenotypes (Additional file 2: Table S2). Combined, significant larval locomotor defects were observed for 58 genes in either ubiquitous or neuronal RNAi; 21 genes showed defects in both types of RNAi. It should be noted that lack of a phenotype could be a consequence of inadequate knockdown.

Several data suggested the validity of this RNAi screen. We randomly selected one gene, Goo, and tested its neuronal RNAi using a UAS-RNAi strain from the VDRC stock center. This strain showed a phenotype highly similar to that of the UAS-RNAi strain from the Bloomington stock center (Fig. 1b), suggesting that the phenotype is gene specific. In addition, among the genes with RNAi larval locomotor phenotypes, mutants of $c a c$, CanA1, Caps, dys, and norpA are known to have locomotor defects in adults (FlyBase). The consistency between our RNAi results with known locomotor effects of chromosomal mutations suggested that the RNAi effects are likely gene specific.

The sensitivity of our phenotyping is high because of the quantitative measurements. Among the parameter values that we found to be significantly different than wild-type values, most of them were $0.5-2$ standard deviations (SDs) away from wild-type values (Fig. 1c). $91 \%$ of these 58 genes identified with larval locomotor defects in this study have not been previously associated with any locomotor defects. Among the 68 genes for which we had locomotor profiles, only 6 genes were annotated with locomotor defects with any mutant allele at any developmental stage in FlyBase (flybase.org, version FB2016_05). Our phenotyping data recaptured 5 of the 6 genes to have significant larval locomotor defects, and detected 53 more genes with such phenotype. Together, these data suggested that our current knowledge on genetic control of larval locomotion is still largely incomplete, and that our method is highly effective in discovering genes with such functions.

\section{Neuronal genes required for larval locomotion}

To understand how genes function in the neurons to regulate larval locomotion, we seek answers to two questions: what genes are involved, and how these genes interact.

RNAi of the following 42 genes in the neurons caused significant larval locomotor defects: Ank2, Arf79F, bru-3, cac, CanA-14F, CanA1, Caps, CG18208, CG31140, CngB,

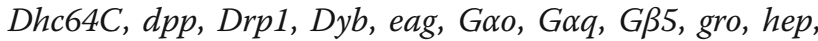
lin-28, Liprin- $\alpha$, Med, nAChR $\alpha 1, n A C h R \alpha 3, n A C h R \alpha 4$, nAChRa6, nSyb, Rab27, Rab3, Rab3-GEF, Rab6, Rdl, retn, RhoGAP100F, sei, Snap24, ss, Syb, trio, unc-13, $X 11 L$ (Fig. 2a, Additional file 2: Table S2). Among them, an interesting case is the nicotinic acetylcholine receptors (nAChRs). Mutations in human nAChRs are linked to a range of diseases such as epilepsy and autoimmune diseases [23]. There are $10 \mathrm{nAChRs}$ in the Drosophila genome, $\alpha 1-\alpha 7$ and $\beta 1-\beta 3$ [24]. These nAChRs are expressed in the nervous system and are targets of insecticides [24]. Their in vivo physiological functions are largely unknown except that $\alpha 7$ is required for an escape behavior [25].

Eight nAChRs, $\alpha 1-\alpha 7$ and $\beta 2$, have RNAi strains available at the Bloomington center and were tested in this study. Neuronal inactivation of $\alpha 1$ and $\alpha 3$ produced most severe and very similar larval locomotor phenotypes (Fig. 2b). It was reported that mutants of $n A C h R \alpha 1$ and $n A C h R \beta 2$ showed resistance to the neonicotinoid class of insecticides [26]. Consistent with this, neuronal RNAi of $n A C h R \beta 2$ had a larval locomotor profile highly similar to that of $n A C h R \alpha 1$, although the phenotype of $n A C h R \beta 2$ is weaker than that of $n A C h R \alpha 1$ (Fig. 2b). These data indicated that among the nAChRs, $\alpha 1, \alpha 3$ and $\beta 2$ share similar functions in regulating larval locomotive behaviors. It was shown that $n A C h R \alpha 5$ and $n A C h R \alpha 7$ can form heteromeric ion channels [27]. It is possible that $\alpha 1, \alpha 3$ and $\beta 2$ may also form heteromeric ion channels.

\section{Interactions of neuronal locomotor genes}

To identify the interactions among the 42 neuronal larval locomotive genes, we computed the Pearson correlation 

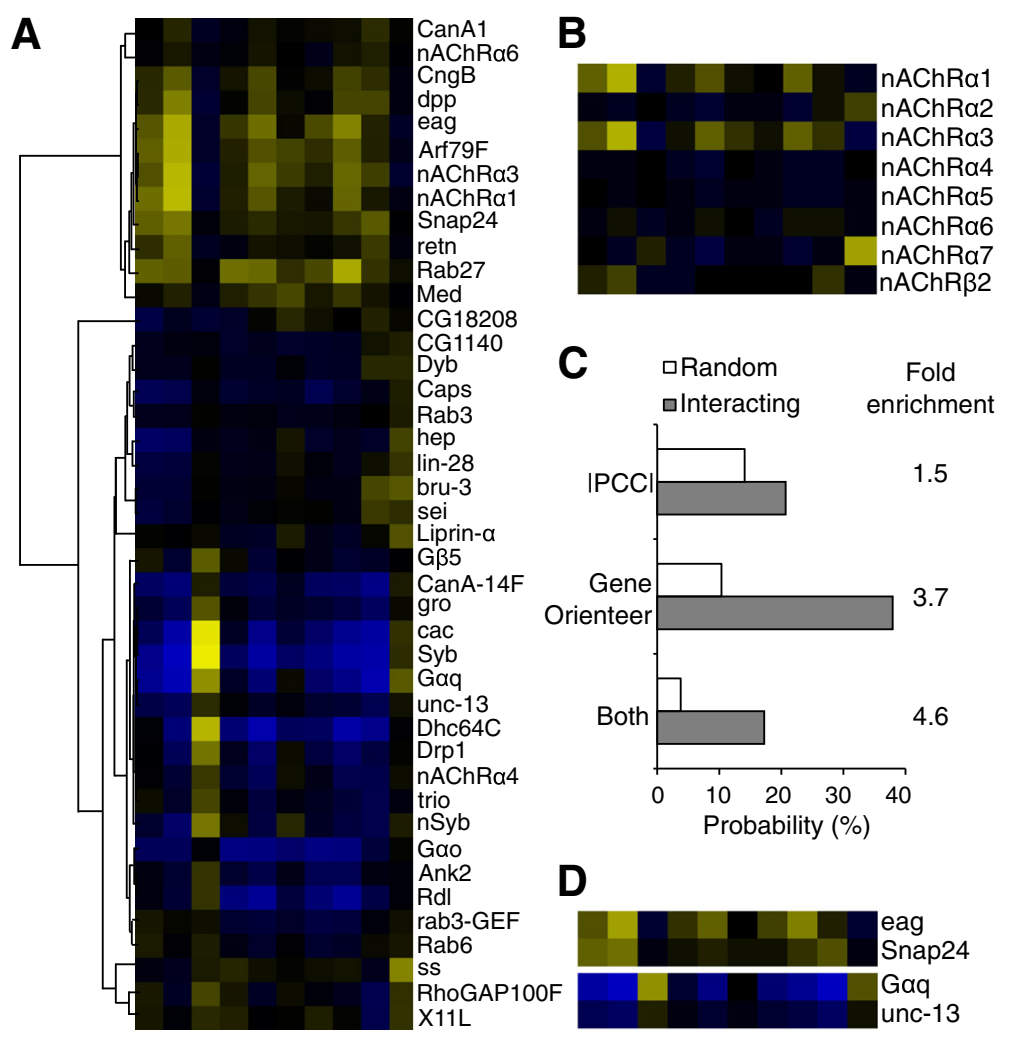

Fig. 2 Genes required in the neurons to regulate locomotor behaviors. a Hierarchical clustering of the 41 neuronal profiles with significant phenotypes. $\mathbf{b}$ Neuronal profiles of nAChRs. $\mathbf{c}$ Known interacting genes are enriched with correlated profiles $(|\mathrm{PCC}|>0.7)$ and high GeneOrienteer scores. $\mathbf{d}$ Neuronal profiles of the four genes that have high $\mid \mathrm{PCC}$ and GeneOrienteer scores with nAChRa1 and nAChRa3

coefficient (PCC) to evaluate the similarity of phenotypic patterns. We reasoned that interacting gene pairs were more likely to have similar (PCC close to 1 ) or opposite (PCC close to -1 ) phenotypic patterns, resulting a high absolute value of PCC (|PCC $\mid$ close to 1$)$. In contrast, if two phenotypic patterns were not correlated at all (|PCC| close to 0 ), then less likely these two genes would interact. To verify such rationale, we queried the databases BioGrid (thebiogrid.org) and FlyBase for known interactions. Among all genes we tested, there were 29 gene pairs known to interact genetically or physically. Indeed, these interacting genes were more likely to have high $|\mathrm{PCC}|$ values of their locomotive profiles. $21 \%$ of interacting gene pairs had $|\mathrm{PCC}|$ value over 0.7 for their locomotive profiles, whereas only $14 \%$ of random parings of neuronal profiles did (Fig. 2c), confirming that $|\mathrm{PCC}|$ could be used to distinguish interacting genes. The 42 neuronal larval locomotive genes generated $861(42 \times 41 / 2=861)$ pairwise combinations. Among them, a total of 302 pairs showed $|\mathrm{PCC}|$ above 0.7 .

To further prioritize these 302 gene pairs for genetic interactions, we queried GeneOrienteer (geneorienteer.org), a database that predicts genetic interactions by integrating expression, phenotype, gene ontology, and interaction data from multiple species [28]. Our previous investigation of C. elegans locomotive genes showed genetic interactions were most enriched among gene pair that satisfied two conditions: $|\mathrm{PCC}|$ above 0.7 , and GeneOrienteer score over 4 [14]. A similar result was observed in this study of fly genes: interacting pairs were 4.6 times more likely than random pairing of locomotor profiles to satisfy both criteria, while individual criteria could only provide 1.5 and 3.7 times enrichment (Fig. 2c). Two hundred twentytwo gene pairs among the 42 neuronal larval locomotive genes had GeneOrienteer scores over 4 . Using both $|\mathrm{PCC}|$ and GeneOrienteer scores as criteria, 81 pairs of neuronal larval locomotive genes were identified as high-confidence interactions (Additional file 3: Table S3, Fig. 3).

Some of these 81 high-confidence interactions are consistent with known interaction data. For example, the gene products of two pairs, $u n c-13-S y b$ and Snap24 - Syb, have been shown to interact physically in yeast two-hybrid experiments (BioGrid). The yeast orthologous pair of Arf79F - Syb and the human orthologous pair of Snap24 - unc-13 also encode proteins that physically interact (BioGrid). The orthologs of 13 pairs interact genetically in C. elegans, in-

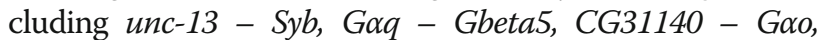

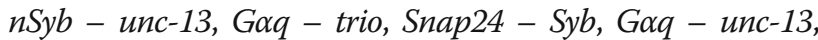




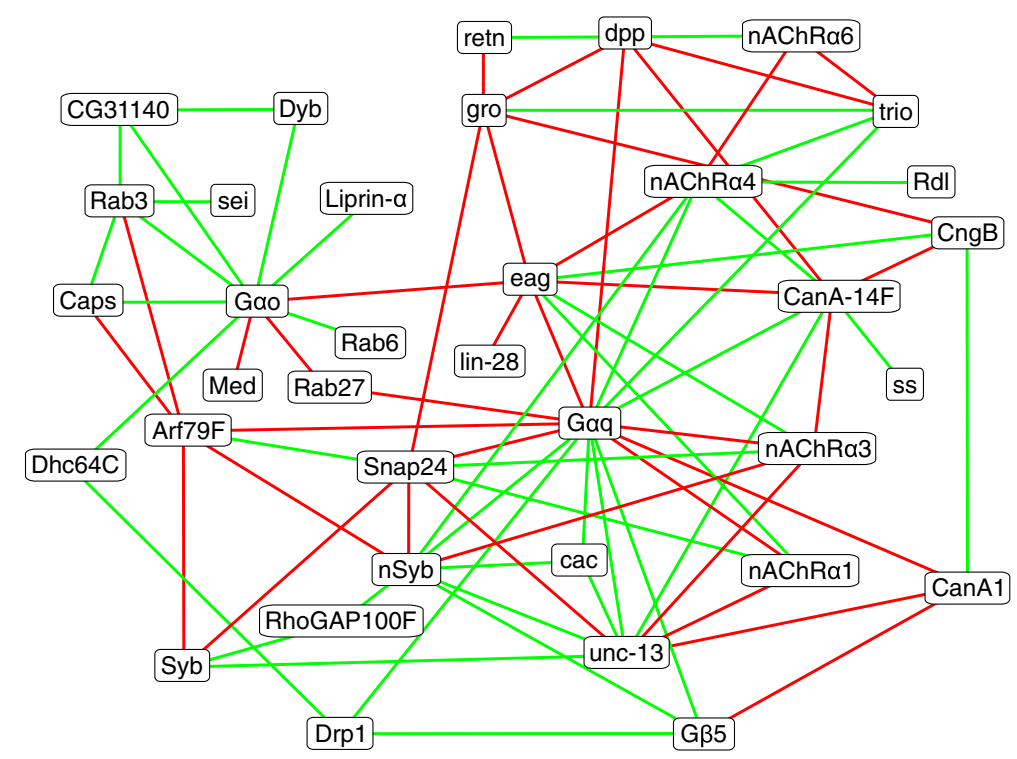

Fig. 3 High-confidence neuronal gene interactions. Green edges indicate two genes have similar behavioral profiles; red edges indicate two genes have opposite behavioral profiles

CanA-14F - Goq, CanA1 - Goq, Caps - Goo, Goo Liprin- $\alpha, n$ Syb - RhoGAP100F, RhoGAP100F - Syb (WormBase). These data demonstrated the validity of these high-confidence interactions.

The high-confidence interaction network shows that $n A C h R \alpha 1$ and $n A C h R \alpha 3$ interact with the SNARE protein Snap24, and the potassium channel eag, with similar phenotypic patterns; $n A C h R \alpha 1$ and $n A C h R \alpha 3$ also interact with the synaptic vesicle protein $u n c-13$, and the G protein G $\alpha q$, with opposite phenotypic patterns (Figs. 2d, 3). It was reported that unc-13 functions downstream of $G \alpha q$ signalling, and upstream of vesicular fusion at the neuromuscular junction (NMJ) $[29,30]$. Our data suggested that eag and nAChRs have an antagonistic effect on this Goq signalling pathway in locomotor behaviors. The involvement of Snap24 suggested that the antagonistic effect on Goq signalling is possibly mediated through neurotransmitter release or reuptake.

\section{Site-of-action for larval locomotive genes}

To understand whether the locomotor genes primarily function in neurons or in additional tissues, we compared the locomotive profiles from pan-neuronal RNAi with those from ubiquitous RNAi. If inactivating the gene in the neurons generates the same phenotype as inactivating the gene ubiquitously, then the gene function is most likely to be neuronal. As RNAi strength varies with different Gal4 drivers, the phenotypic severity of different RNAi may differ, causing difference in our parameter values. Therefore, we evaluated the PCC of profiles because phenotypic patterns are less affected by RNAi strength.
Pan-neuronal RNAi profiles were more likely to be correlated with ubiquitous RNAi profiles of the same genes. $26 \%$ genes had highly correlated (PCC $>0.7$ ) ubiquitous and neuronal profiles; among genes with significant locomotor phenotypes in both neuronal and ubiquitous RNAi, $38 \%$ had PCC $>0.7$ for ubiquitous and neuronal profiles (Fig. 4a). These data suggested that many genes we tested predominantly function in the neurons. For example, Arf79F, CG31140, Drp1, eag, Goo, nAChRa3, Rab 27, and ss, had highly correlated ubiquitous and neuronal profiles (Fig. 4b), suggesting that these genes function primarily in the neurons to regulate locomotion. In contrast, $G \beta 5$, lin28, Liprin- $\alpha$, Med, rab3-GEF, retn, RhoGAP100F, had little correlation (PCC $<0.1)$ between their ubiquitous and neuronal profiles (Fig. 4c), suggesting that these genes have functions in non-neuronal tissues as well as neurons to regulate larval locomotion.

For the genes with severe neuronal phenotypes, we investigated whether they primarily function in motor neurons. The behavioral profiles from the pan-neuronal elav-Gal4 driver were compared with those from the motor-neuronal D42-Gal4 driver. Arf79F, eag, nAChR 1 , $n A C h R \alpha 3$, and $R d l$ showed highly correlated $(\mathrm{PCC}>0.7)$ pan-neuronal and motor-neuronal profiles (Fig. 4d), suggesting that these genes primarily function in the motor neurons to regulate larval locomotion. Drp1, G $\alpha o, G \alpha q$ showed moderately correlated (PCC between 0.6-0.7) pan-neuronal and motor-neuronal profiles. These genes may still primarily function in the motor neurons. Cac, CanA1, and unc-13 had uncorrelated (PCC < 0.3) panneuronal and motor-neuronal profiles (Fig. 4e), suggesting that these genes have functions in neurons other 

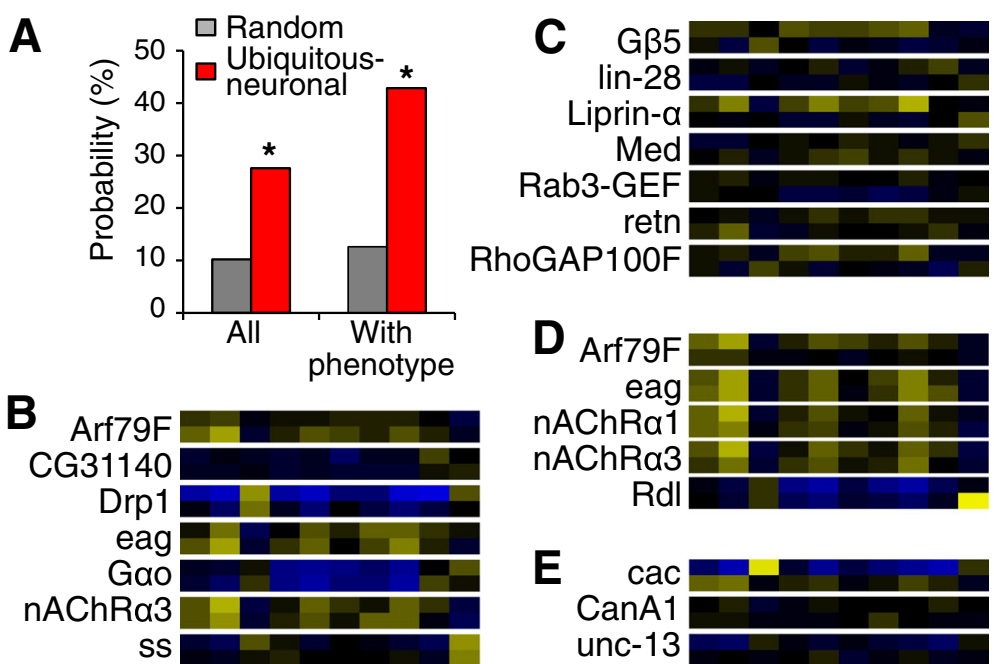

Fig. 4 Locomotor genes site-of-action. a The ubiquitous and neuronal profiles from the same gene is more likely to have PCC > 0.7 than randomly paired profiles. In one data set all profiles were tested; in the other, only profiles with significant phenotypes were tested. * $p<0.01$, Fisher's exact test. b Genes with correlated (PCC > 0.7) ubiquitous (top) and pan-neuronal (bottom) behavioral profiles. c Genes with uncorrelated (PCC < 0.1) ubiquitous (top) and pan-neuronal (bottom) behavioral profiles. $\mathbf{d}$ Genes with correlated pan-neuronal (top) and motor-neuronal (bottom) behavioral profiles. e Genes with uncorrelated pan-neuronal (top) and motor-neuronal (bottom) behavioral profiles

than motor neurons. The requirement of different neurons in larval locomotor regulation is consistent with previous findings such as $[8,31]$.

For the nAChRs, $n A C h R \alpha 1$ and $n A C h R \alpha 3$ showed highly similar phenotypic profiles with pan-neuronal, and motor-neuronal RNAi (Fig. 4d), suggesting that these genes primarily function in motor neurons. Similarly, their interacting genes, $G \alpha q$ and $e a g$, are also likely to primarily function in motor neurons (Fig. 4d). In contrast, the interactor $u n c-13$ is likely to function in other neurons and non-neuronal tissues (Fig. 4c, e).

\section{Conservation of gene functions in locomotor phenotypes and neuronal expression}

Because all genes we tested in this study were orthologs of C. elegans genes that had been analyzed previously for their locomotor defects [14], such data enabled us to compare gene functions in C. elegans and D. melanogaster to understand their levels of conservation. Three aspects of gene functions were evaluated: expression, phenotype, and gene interactions. The two animals have different nervous system anatomy and different locomotive pattern: $C$. elegans employs a sinusoidal wave of crawling whereas fly larvae employ peristalsis crawling. Therefore, the detailed cellular gene expression pattern and locomotive parameters are not comparable between the two species. However, we can compare gene function at a higher level such as whether the genes are neuronally expressed and whether they affect movement.
All orthologous worm genes were annotated as neuronal genes in WormBase. We queried FlyBase and found that $67 \%(46 / 69)$ genes were also annotated as neuronally expressed, i.e., associated with terms such as brain, nerve, neuron, or nervous system. In contrast, only $15 \%$ of all genes in the fly genome and 19\% of all fly genes with worm orthologs were annotated as expressed in the neurons (Fig. 5a). In addition to FlyBase annotations, 12 genes that were not annotated in FlyBase as neuronally expressed showed locomotor phenotypes in this study when inactivated in the neurons, suggesting that they function in the neurons. Combined, 87\% (60/69) of our tested fly genes are neuronal genes (Fig. 5a), demonstrating a high level of conservation in gene expression.

In addition to expression, these genes also showed a high level of conservation in phenotypes. All orthologous worm genes had mutants with significant locomotor defects [14]. In this study, upon RNAi inactivation, 85\% (59/69) of our tested fly genes showed locomotor phenotypes (Fig. 5b). We further queried FlyBase to estimate how likely a randomly selected gene would display a locomotor phenotype. Among FlyBase phenotype annotations, only $3.1 \%$ of all genes in the fly genome and $2.5 \%$ of all genes with worm orthologs were annotated with locomotor defects (Fig. 5b). This ratio significantly ( $p<0.01$, Fisher's exact test) increased to $10 \%$ among our tested genes (Fig. 5b), demonstrating that locomotor defects are enriched among these genes. Together, these data suggested a strong conservation among these genes in locomotor phenotypes. 


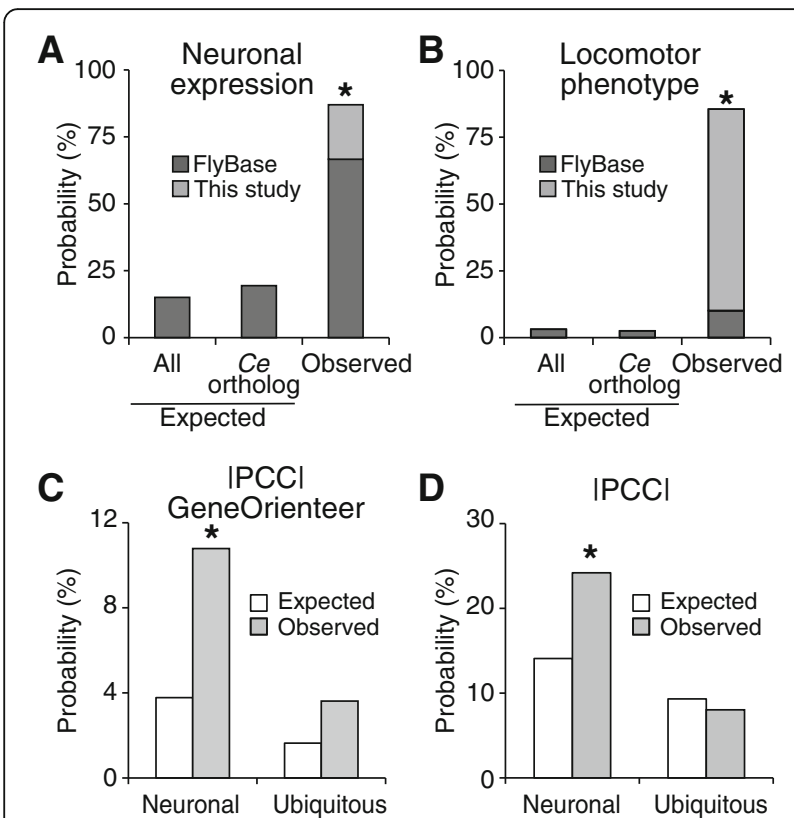

Fig. 5 Conservation and rewiring of genetic control of locomotor behaviors between worms and flies. a The examined set was enriched with genes expressed in the neurons. $\mathbf{b}$ The examined genes were enriched with locomotor phenotypes. In (a) and (b), expected background values were computed using Flybase annotations for all genes (All) or genes with C. elegans orthologs (Ce ortholog). ${ }^{*}, p<0.01$ comparing observed value (FlyBase alone, or combined data from FlyBase and this study) with both expected values, Fisher's exact test. $\mathbf{c}$, d Mild conservation of genetic interactions, if interaction was defined using both $|P C C|$ for locomotive profiles and high GeneOrienteer scores (c) or only |PCC| values (d). Both neuronal and ubiquitous profiles were analyzed. Observed values were computed using fly gene pairs with interacting worm orthologous pairs. Expected values were computed using all combinations of genes. ${ }^{*}$ indicates $p<0.01$ comparing observed and expected values, Fisher's exact test

Rewiring of genetic interactions among locomotor genes Next we examined the conservation of genetic interactions. Worm interacting gene pairs were defined as genes with $|\mathrm{PCC}|>0.7$ for worm locomotive profiles and GeneOrienteer score over 4 [14]. For fly gene pairs whose worm orthologous pairs were interacting, 11\% were interacting if we used the same criteria for fly gene interactions and if we used locomotive profiles from neuronal RNAi (Fig. 5c). While such conservation level was drastically lower than those in expression or phenotype ( 11 vs. $\geq 85 \%$ ), it is significantly ( $p<0.001$, Fisher's exact test) higher than the expected probability from random gene pairs (11 vs. 4\%, Fig. 5c). In comparison with neuronal locomotive profiles, profiles from ubiquitous RNAi showed even a lower level of conservation: Fly gene pairs with interacting worm orthologous pairs had only $2.4 \%$ probability of interacting, which was not significantly higher than the $1.2 \%$ from random pairs (Fig. $5 \mathrm{c}$ ).
We investigated whether our results on genetic interactions were biased by the GeneOrienteer algorithm as it used orthologous data. When we eliminated GeneOrienteer scores and used $|\mathrm{PCC}|$ values as the sole criterion for interaction, we observed that $24 \%$ of genetic interactions were conserved if neuronal profiles were used, which was significantly higher than the expected $14 \%$ from random pairs (Fig. 5d). Conservation of genetic interactions was not observed in ubiquitous profiles (Fig. 5d). These data confirmed that the conservation level of genetic interactions was much lower than those of expression and phenotype.

\section{Motor-neuronal control of body length}

While we used only 10 locomotive parameters in this study, our imaging system extracts $20+$ parameters from the videos. One parameter that was measured by the system but not used in the locomotive profiles was body size. As male larvae are smaller than female larvae, we analyzed them separately in all body length measurements. Notably, inactivation of two genes, $R d l$ and $G \alpha o$, in neurons caused significantly reduced larval body size in both females and males (Fig. 6a).

As neuronal RNAi of $R d l$ and Goo also caused severe locomotive defects, it seemed possible that the reduced body length might be a secondary defect of the locomotive phenotypes. For example, if a larva could not move well, it might not reach food well, and thus could have a small body length because of malnutrition. This was unlikely under our culture conditions as the larvae were maintained continuously on food. Furthermore, neuronal RNAi of many genes caused severe locomotor defects, without affecting body length. For example, neuronal RNAi of $s y b$ reduced the larval crawling speed to a level similar to $R d l$ and $G \alpha o$ (Fig. 6b), yet caused no body length defects (Fig. 6a). Therefore, we hypothesize that the reduced larval length of $R d l$ and $G \alpha o$ reflects a primary defect in gene function.

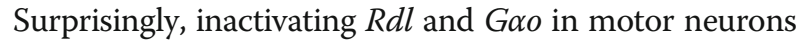
using the D42-Gal4 driver reproduced the shortenedbody-length phenotype (Fig. 6c). In contrast, inactivating these genes in insulin producing brain cells using a dilp2Gal4 driver did not cause reduced body length (Fig. 6c). These data suggested that there is a motor-neuronal control of body length in D. melanogaster, and that such control requires the function of $R d l$ and $G \alpha o$ in motor neurons.

\section{Database}

In addition to the supplementary tables, an interactive database was built to enable queries of videos and behavioral profiles including all $20+$ parameters. The database provides raw measurements as well as normalized measurements of each animal for query and download. 


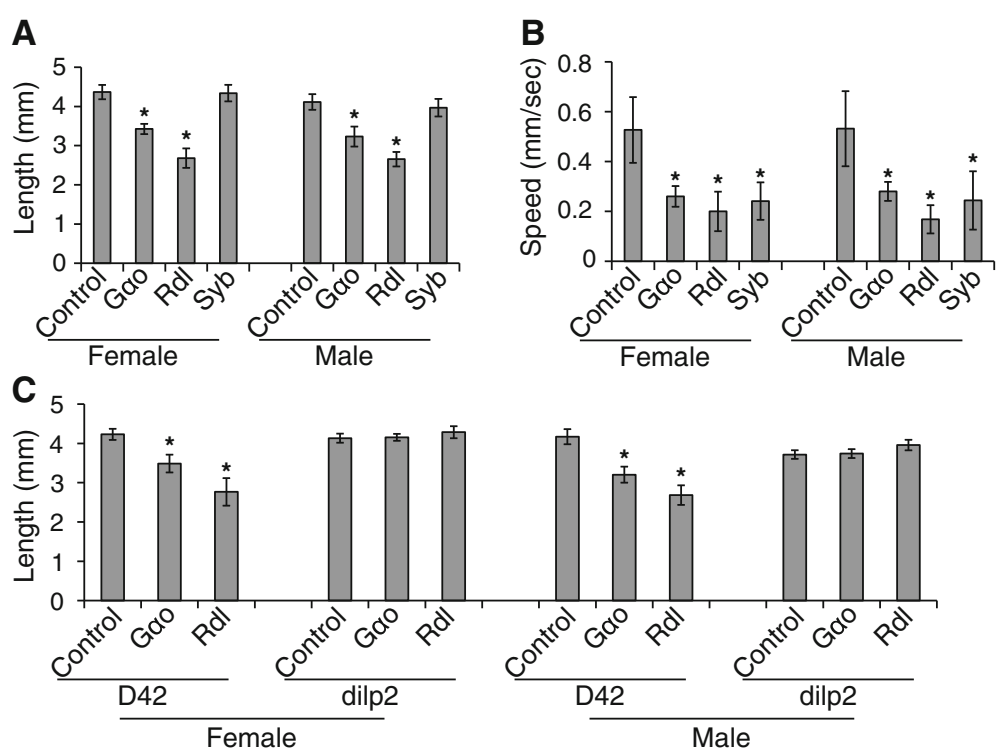

Fig. 6 Motor neuronal control of body size. a RNAi of Gao and Rdl, but not Syb, caused smaller body size in both males and females. $\mathbf{b}$ The locomotor defects are similarly severe for neuronal RNAi of Gao, Rdl, and Syb. c RNAi of Gao and Rdl in motor neurons (D42-Gal4 driver), but not insulin-producing neurons (dilp2-Gal4 driver) recaptured the smaller body size phenotype. *, $p<0.01$, Student's t-test. $n \geq 10$ animals for each genotype. Bars and error bars indicate mean and standard deviation

The database can be accessed at http://wormloco.org, the same website that also stores C. elegans locomotor behavioral profiles [14].

\section{Discussion}

We present here the high-content larval locomotive profiles of 69 genes whose C. elegans orthologs are neuronal signalling genes with locomotor phenotypes. Profiles were collected after inactivation of these genes by RNAi in all cells, neurons, and motor neurons. Bioinformatic analysis on these profiles revealed 42 genes whose functions are required in the neurons to maintain normal larval locomotor behaviors, and 81 high-confident interactions among these genes. While many of these genes are known to be expressed in neurons and have adult behavioral phenotypes, the difference between our study and the previous findings are: 1) this study revealed the functions of these genes in larval instead of adult behaviors; 2) this study provided quantitative, high-content phenotypic measurements instead of qualitative observations or single-metric measurements.

Using the nAChR family as an example, we showed how such analysis brings insights to gene functions. Our profiles revealed that inactivation of $n A C h R \alpha 1$ and $n A C h R \alpha 3$ in the neurons caused severe movement disorders. The interaction analysis suggested that these two nAChRs function similarly with Snap24 and eag, but antagonistically with $G \alpha q$ and $u n c-13$. Site-of-action analysis suggested that $n A C h R \alpha 1, n A C h R \alpha 3$, eag, Goq function primarily in the motor neurons. These results are consistent with previous reports that $G \alpha q$ and $u n c-13$ function in the same signalling pathway in NMJ $[29,30]$. It was reported that cholinergic input directly stimulates motor neurons [32], consistent with our finding that nAChRs are required in the motor neurons to maintain normal movement.

While high-content profiling cannot definitively prove genetic interactions as epistasis analysis, the effectiveness of this method has been well established [11-14]. As there is no high-throughput method to epistasis analysis in flies, experimental validation of these predicted genetic interactions is difficult. We thus relied on a computational validation of the predicted genetic interactions, and showed that the predicted interactions are enriched among the known positives (known interactions) than negatives (random gene pairs). Random gene pairs were used as negatives because there is no sizable data set on genes that do not interact, and because genetic interactions are sparse among all gene pairs.

It has remained unknown whether genetic interaction networks are conserved for multicellular organisms. This is mostly due to lack of large-scale genetic interaction studies: the only available multicellular in vivo genetic interaction networks are synthetic lethality networks in C. elegans [33, 34]. While certain conservation was observed for genetic interaction networks between budding and fission yeast, no conservation was observed between these worm networks and the yeast ones [35, 36]. Using the same methodology of high-content profiling, we provided comparable data sets to estimate the conservation 
of genetic interaction networks in worms and flies. Similar to the worm-yeast result, no strong conservation of genetic interactions between worms and flies was observed under ubiquitous RNAi. C. elegans and Drosophila larvae have different nervous system and muscle anatomy. As genetic interactions can involve genes functioning in different cells, the difference in cellular circuitry may explain the lack of conservation. Similar reasoning may also explain the mild conservation between worm and fly neuronal genetic interactions $(11 \%$ conservation rate, Fig. 4d), as these interactions are limited to the same tissue. Consistent with this hypothesis, this mild conservation rate is similar to the genetic interaction conservation rate observed between budding yeast $S$. cerevisiae and fission yeast S. pombe: $17 \%$ negative interactions and $10 \%$ positive interactions were conserved [36].

The observed motor neuronal control of body size was unexpected. One possible mechanism for such motorneuronal effect of body size is through muscle cells. It was discovered that inhibition of insulin receptor ( InR) in muscle cells could cause systemic effects and reduce the body size of the entire larvae [37] an effect that might be mediated at least in part by the Foxodependent increase in release of ImpL2, an insulin signalling inhibitor [38]. It is possible that $R d l$ and $G \alpha o$ functions in motor neurons affect the muscle state, which subsequently changes the activities of these regulators in muscle cells, and impacts body size. Given that $R d l$ encodes the Drosophila $\mathrm{GABA}_{\mathrm{A}}$ receptor, which mediates inhibitory synaptic transmission, the $R d l$ knockdown is expected to increase motor neuron activity and hence synaptic input onto the muscle; this increased synaptic input might activate muscle Foxo. Consistent with this model, it was reported previously that effects of altered synaptic transmission in Drosophila larvae on activity of muscle insulin signalling components [39].

It was shown that a coordinated action from both excitatory neurons, which release acetylcholine, and inhibitory neurons, which release GABA, is required to generate the sequential firing of motor neurons for the peristaltic movement [40]. The acetylcholine receptor (nAChR) and the $\mathrm{GABA}_{\mathrm{A}}$ receptor (Rdl) are likely to function in different dendrites of motor neurons to mediate the excitatory and inhibitor synaptic transmission, respectively, to drive the rhythmic firing of motor neurons responsible for peristaltic movements.

We do not know whether motor neuronal control of body size also exists in C. elegans. Neuronal control of body size exists in $C$. elegans, as it was reported that gene functions in sensory neurons affect body size in $C$. elegans [41]. Mutants of the C. elegans orthologs of $R d l$ and Goo, unc-49 and goa-1, had a smaller body length (wormloco.org). However, the site-of-action for these genes in size-regulation is unknown in C. elegans.

\section{Conclusion}

Our high-content profile data provided a framework for understanding the genetic control of larval locomotion. In addition to providing clues for individual genes functions, such system-level approach also enabled evaluation of conservation and rewiring of genetic interaction networks.

\section{Additional files}

Additional file 1: Table S1. Genes studied. List of the gene names, FlyBase IDs of the genes, the Bloomington stock numbers of fly strains used, and the worm orthologs of the fly genes (XLSX $12 \mathrm{~kb}$ ).

Additional file 2: Table S2. Locomotive profiles of genes with significant phenotypes. Profiles are composed of normalized values for ten locomotive parameters. Profiles for both neuronal and ubiquitous RNAi are listed. The summary page lists genes with significant phenotypes (XLSX $26 \mathrm{~kb}$ ).

Additional file 3: Table S3. Genetic interactions inferred from locomotive profiles. Predicted genetic interactions with $|\mathrm{PCC}|>0.7$ and GeneOrienteer score over 4 (XLSX 12 kb)

\section{Abbreviations}

FDR: False discovery rate; InR: Insulin receptor; nAChR: nicotinic acetylcholine receptor; NMJ: Neuromuscular junction; PCC: Pearson correlation coefficient; SD: Standard deviation

\section{Acknowledgement}

We thank José Garza Martínez and Joaquina Nunez for technical assistance.

\section{Funding}

This research was funded by the National Institutes of Health (HG004724, DA018341); Department of Defense Office of Congressionally Directed Medical Research (W81XWH-16-1-0110); and by a Searle Scholar grant to WZ.

Availability of data and materials

All relevant data are available within the manuscript.

\section{Authors' contributions}

MS, WZ conceived and designed the experiments. BAM, MLC, OPR performed experiments. WZ and BAM analyzed the data. WZ wrote the paper. All authors read and approved the final manuscript.

Competing interests

The authors declare that they have no competing interests.

Consent for publication

Not applicable.

Ethics approval and consent to participate Not applicable.

\section{Publisher's Note}

Springer Nature remains neutral with regard to jurisdictional claims in published maps and institutional affiliations.

Received: 16 January 2017 Accepted: 9 May 2017

Published online: 12 May 2017

\section{References}

1. Soll DR. The use of computers in understanding how animal cells crawl. Int Rev Cytol. 1995;163:43-104

2. Caldwell JC, Miller MM, Wing S, Soll DR, Eberl DF. Dynamic analysis of larval locomotion in Drosophila chordotonal organ mutants. Proc Natl Acad Sci. 2003;100:16053-8. 
3. Wang JW, Soll DR, Wu C-F. Morphometric description of the wandering behavior in Drosophila larvae: a phenotypic analysis of K+ channel mutants. J Neurogenet. 2002;16:45-63.

4. Wang JW, Sylwester AW, Reed D, Wu DA, Soll DR, Wu CF. Morphometric description of the wandering behavior in Drosophila larvae: aberrant locomotion in $\mathrm{Na}+$ and $\mathrm{K}+$ channel mutants revealed by computer-assisted motion analysis. J Neurogenet. 1997;11:231-54.

5. Heckscher ES, Lockery SR, Doe CQ. Characterization of Drosophila larval crawling at the level of organism, segment, and somatic Body Wall musculature. J Neurosci. 2012:32:12460-71.

6. Berni J, Pulver SR, Griffith LC, Bate M. Autonomous circuitry for substrate exploration in freely moving Drosophila larvae. Curr Biol. 2012;22:1861-70.

7. Ohyama T, Jovanic T, Denisov G, Dang TC, Hoffmann D, Kerr RA, et al. Highthroughput analysis of stimulus-evoked Behaviors in Drosophila Larva reveals multiple modality-specific escape strategies. PLoS One. 2013;8:e71706.

8. Vogelstein JT, Park Y, Ohyama T, Kerr RA, Truman JW, Priebe CE, et al. Discovery of brainwide neural-behavioral maps via multiscale unsupervised structure learning. Science. 2014;344:386-92.

9. Jovanic T, Schneider-Mizell CM, Shao M, Masson J-B, Denisov G, Fetter RD, et al. Competitive Disinhibition mediates behavioral choice and sequences in Drosophila. Cell. 2016;167:858-870.e19.

10. Mackay TFC. Epistasis and quantitative traits: using model organisms to study gene-gene interactions. Nat Rev Genet. 2014;15:22-33.

11. Green RA, Kao H-L, Audhya A, Arur S, Mayers JR, Fridolfsson HN, et al. A high-resolution C. elegans essential gene network based on phenotypic profiling of a complex tissue. Cell. 2011;145:470-82.

12. Sönnichsen B, Koski LB, Walsh A, Marschall P, Neumann B, Brehm M, et al. Full-genome RNAi profiling of early embryogenesis in Caenorhabditis elegans. Nature. 2005;434:462-9.

13. Brown AEX, Yemini El, Grundy LJ, Jucikas T, Schafer WR. A dictionary of behavioral motifs reveals clusters of genes affecting Caenorhabditis elegans locomotion. Proc Natl Acad Sci U S A. 2013;110:791-6.

14. Yu H, Aleman-Meza B, Gharib S, Labocha MK, Cronin CJ, Sternberg PW, et al. Systematic profiling of Caenorhabditis elegans locomotive behaviors reveals additional components in G-protein Gaq signaling. Proc Natl Acad Sci U S A. 2013;110:11940-5.

15. Aleman-Meza B, Jung S-K, Zhong W. An automated system for quantitative analysis of Drosophila larval locomotion. BMC Dev Biol. 2015;15:11.

16. Yeh E, Gustafson K, Boulianne GL. Green fluorescent protein as a vital marker and reporter of gene expression in Drosophila. Proc Natl Acad Sci U S A. 1995;92:7036-40.

17. Luo L, Liao YJ, Jan LY, Jan YN. Distinct morphogenetic functions of similar small GTPases: Drosophila Drac1 is involved in axonal outgrowth and myoblast fusion. Genes Dev. 1994;8:1787-802.

18. Rulifson EJ, Kim SK, Nusse R. Ablation of insulin-producing neurons in flies: growth and diabetic phenotypes. Science. 2002;296:1118-20.

19. Benjamini $Y$, Hochberg $Y$. Controlling the false discovery rate: a practical and powerful approach to multiple testing. J R Stat Soc Ser B (Methodological). 1995;57:289-300.

20. de Hoon MJL, Imoto S, Nolan J, Miyano S. Open source clustering software. Bioinforma Oxf Engl. 2004;20:1453-4.

21. Saldanha AJ. Java Treeview-extensible visualization of microarray data Bioinforma Oxf Engl. 2004;20:3246-8.

22. Ni J-Q, Zhou R, Czech B, Liu L-P, Holderbaum L, Yang-Zhou D, et al. A genome-scale shRNA resource for transgenic RNAi in Drosophila. Nat Methods. 2011;8:405-7.

23. Jones AK, Sattelle DB. Diversity of insect nicotinic acetylcholine receptor subunits. Adv Exp Med Biol. 2010;683:25-43.

24. Jones AK, Brown LA, Sattelle DB. Insect nicotinic acetylcholine receptor gene families: from genetic model organism to vector, pest and beneficial species. Invertebr Neurosci IN. 2007;7:67-73.

25. Fayyazuddin A, Zaheer MA, Hiesinger PR, Bellen HJ. The nicotinic acetylcholine receptor Dalpha7 is required for an escape behavior in Drosophila. PLoS Biol. 2006;4:e63.

26. Perry T, Heckel DG, McKenzie JA, Batterham P. Mutations in Dalpha1 or Dbeta2 nicotinic acetylcholine receptor subunits can confer resistance to neonicotinoids in Drosophila melanogaster. Insect Biochem Mol Biol. 2008;38:520-8.

27. Lansdell SJ, Collins T, Goodchild J, Millar NS. The Drosophila nicotinic acetylcholine receptor subunits Da5 and Da7 form functional homomeric and heteromeric ion channels. BMC Neurosci. 2012;13:73.
28. Zhong W, Sternberg PW. Genome-wide prediction of C. elegans genetic interactions. Science. 2006:311:1481-4.

29. Aravamudan B, Fergestad T, Davis WS, Rodesch CK, Broadie K. Drosophila UNC-13 is essential for synaptic transmission. Nat Neurosci. 1999:2:965-71.

30. Aravamudan B, Broadie K. Synaptic Drosophila UNC-13 is regulated by antagonistic G-protein pathways via a proteasome-dependent degradation mechanism. J Neurobiol. 2003;54:417-38.

31. Clark MQ, McCumsey SJ, Lopez-Darwin S, Heckscher ES, Doe CQ. Functional genetic screen to identify Interneurons governing Behaviorally distinct aspects of Drosophila larval motor programs. G3 GenesGenomesGenetics. 2016;6:2023-31.

32. Baines RA, Bate M. Electrophysiological development of central neurons in the Drosophila embryo. J Neurosci. 1998;18:4673-83.

33. Lehner B, Crombie C, Tischler J, Fortunato A, Fraser AG. Systematic mapping of genetic interactions in Caenorhabditis elegans identifies common modifiers of diverse signaling pathways. Nat Genet. 2006;38:896-903.

34. Byrne AB, Weirauch MT, Wong V, Koeva M, Dixon SJ, Stuart JM, et al. A global analysis of genetic interactions in Caenorhabditis elegans. J Biol. 2007;6:8.

35. Tischler J, Lehner B, Fraser AG. Evolutionary plasticity of genetic interaction networks. Nat Genet. 2008;40:390-1.

36. Roguev A, Bandyopadhyay S, Zofall M, Zhang K, Fischer T, Collins SR, et al. Conservation and rewiring of functional modules revealed by an epistasis map in fission yeast. Science. 2008:322:405-10.

37. Demontis F, Perrimon N. Integration of insulin receptor/Foxo signaling and dMyc activity during muscle growth regulates body size in Drosophila. Dev Camb Engl. 2009;136:983-93.

38. Owusu-Ansah E, Song W, Perrimon N. Muscle mitohormesis promotes longevity via systemic repression of insulin signaling. Cell. 2013;155:699-712.

39. Penney J, Tsurudome K, Liao EH, Elazzouzi F, Livingstone M, Gonzalez M, et al. TOR is required for the retrograde regulation of synaptic homeostasis at the Drosophila neuromuscular junction. Neuron. 2012;74:166-78.

40. Fushiki A, Zwart MF, Kohsaka H, Fetter RD, Cardona A, Nose A. A circuit mechanism for the propagation of waves of muscle contraction in Drosophila. Elife. 2016:5:e13253.

41. Fujiwara M, Hino T, Miyamoto R, Inada H, Mori I, Koga M, et al. The importance of cGMP Signaling in sensory cilia for body size regulation in Caenorhabditis elegans. Genetics. 2015;201:1497-510.

\section{Submit your next manuscript to BioMed Central and we will help you at every step:}

- We accept pre-submission inquiries

- Our selector tool helps you to find the most relevant journal

- We provide round the clock customer support

- Convenient online submission

- Thorough peer review

- Inclusion in PubMed and all major indexing services

- Maximum visibility for your research

Submit your manuscript at www.biomedcentral.com/submit
) Biomed Central 